\section{Anterior chamber lens implantation in vitrectomised eyes}

\author{
GS Negretti, M Lai, P Petrou, R Walker \\ and D Charteris
}

\begin{abstract}
Purpose To investigate long-term visual acuity (VA) outcomes and complication rates in vitrectomised eyes undergoing anterior chamber intraocular lens (ACIOL) insertion. Patients and methods A single-centre, retrospective case series including all patients who had undergone ACIOL placement at the time of vitrectomy surgery or having had previous vitrectomy, between January 2007 and January 2013. Patients were identified using an electronic database and paper casefile notes were analysed for all patients. Patients were excluded if they had
\end{abstract} $<3$ months follow-up.

Results Two hundred and seventy-one patients were included in the analysis. Mean follow-up was 27 months. One hundred and forty-eight patients were vitrectomised before ACIOL placement. One hundred and twentythree patients underwent vitrectomy at the time of ACIOL placement. Mean bestcorrected visual acuity (BCVA) before ACIOL placement was 1.27 LogMar (SD 0.95). Mean BCVA at final follow-up was 0.51 LogMar (SD 0.66) (paired-sample $T$-test $P<0.001$ ). Forty (15\%) patients developed postoperative cystoid macular oedema (CMO). Eighty-seven $(32 \%)$ patients had an intraocular pressure (IOP) rise acutely post ACIOL insertion. Fifteen $(6 \%)$ patients developed corneal decompensation. Five subsequently required corneal grafting. Sixteen patients had ACIOL displacement or instability requiring further surgery.

Conclusions ACIOL insertion after vitrectomy is effective. Ninety-two per cent of patients maintained or gained VA, comparable to previous studies of ACIOL insertion post complicated cataract surgery. Raised IOP was the most frequent complication: 61 patients were on topical therapy at most recent followup. CMO tended to be acute. Corneal decompensation was infrequent. Eye (2018) 32, 597-601; doi:10.1038/eye.2017.261; published online 8 December 2017

\section{Introduction}

There are three basic strategies for secondary intraocular lens implantation without adequate capsular support: scleral-fixated, iris-fixated, or anterior chamber intraocular lens (ACIOL).

Several, nonprospective, nonrandomised studies with relatively small numbers have compared these alternatives. ${ }^{1-5}$ There remains no consensus as to which approach is the most appropriate choice.

ACIOL insertion is a relatively simple procedure; however, concerns remain about potential complications associated with these lenses. Open-haptic ACIOL designs are known to have lower complication rates than older closed-haptic ACIOLs; ${ }^{6}$ however, risks of corneal decompensation, glaucoma, cystoid macular oedema (CMO), uveitis, and lens instability requiring further surgery remain a concern. Data on the outcomes of ACIOLs in vitrectomised eyes are lacking and outcomes in this situation may differ from nonvitrectomised eyes.

The principal aim of this study was to provide comprehensive data on the risks and benefits of insertion of modern open-haptic ACIOLs in previously vitrectomised eyes. An extensive case series of patients with long follow-up data was utilised.

\section{Materials and methods}

A single-centre retrospective case series study was undertaken with local research committee approval (Registered study number: ROAD 13/013). Patients were eligible for inclusion if they had undergone ACIOL placement at Moorfields Eye Hospital, at the time of vitrectomy surgery or having had had previous vitrectomy under the care of the vitreoretinal service, between January 2007 and January 2013. Case identification was performed using our electronic database. Paper casefile notes were analysed for all patients included in the study. If both eyes had undergone ACIOL insertion,
Department of Vitreoretinal Surgery, Moorfields Eye Hospital, NHS Foundation Trust, London, UK

\section{Correspondence:}

GS Negretti, Department of Vitreoretinal Surgery, Moorfields Eye Hospital, NHS Foundation Trust, 162 City Road, London EC1V 2PD, UK

Tel: +44 (0)20 7253 3411; Fax: +44 (0)20 75662972 E-mail: guynegretti@gmail. com

Received: 23 March 2017 Accepted in revised form: 30 September 2017 Published online: 8 December 2017 
only the first eye was included in the analysis. Visual acuity was recorded as best-corrected visual acuity (BCVA), converted to LogMar, which was either postrefraction or with pinhole correction.

Eyes were excluded if: (a) patients had $<3$ months follow-up post ACIOL insertion or (b) patients had missing paper notes and insufficient information on the electronic patient system to collect their outcome data.

\section{Results}

Three hundred and thirty-three patients were identified as having had a secondary ACIOL inserted. Twenty patients were excluded because their notes were unavailable for analysis and there was not sufficient information about them on our electronic patient system. Forty-two patients were excluded because they had $<3$ months follow-up after their ACIOL insertion. This left 271 patients for analysis: 183 males and 88 females. Total follow-up time ranged from 3 to 100 months with an average follow-up of 27 months. The spread of cases over the study time period was even with no fall in cases towards the later years of the study as illustrated by the fact that 50 patients were included from 2007 and 52 from 2012.

The mean age at operation was 63 years (SD 15.9).

\section{Operation details}

One hundred and forty-eight patients were vitrectomised before ACIOL placement. One hundred and twenty-three patients underwent their primary vitrectomy procedure at the time of ACIOL placement. All patients without a pre-existing peripheral iridectomy (PI) had a surgical PI performed at the time of ACIOL placement. In addition to 123 vitrectomies, additional procedures performed at the time of ACIOL insertion are shown in Table 1.

\section{Visual outcome}

Mean BCVA before ACIOL placement was 1.27 LogMar (SD 0.95). Mean BCVA was significantly better at final follow-up at 0.51 LogMar (SD 0.66) (paired-sample T-test $P<0.001)$. One hundred and seventy-six $(65 \%)$ patients gained >0.2 LogMar visual acuity. Twenty-one (8\%) patients lost $>0.2$ LogMar. Reasons for this loss of vision were documented in 12 patients and are shown in Table 2.

Indications for AC IOL insertion together with mean VA improvement for each indication are shown in Table 3. The different VA outcomes between groups were significant (one-way ANOVA, $P=0.006$ ).

Twenty-one $(46 \%)$ of the subluxed/dislocated crystalline lens cases were caused by trauma and 15 (33\%) were related to Marfan's syndrome. Other less common
Table 1 Additional surgical procedures performed at the time of ACIOL insertion

\begin{tabular}{lc}
\hline Additional procedure & Number of patients \\
\hline Removal of IOL & $108(55 \%)$ \\
Removal of IOL/BVT & $7(4 \%)$ \\
Removal of IOL/preretinal membranectomy & $2(1 \%)$ \\
Removal of IOL/removal of silicone oil & $1(0.5 \%)$ \\
Removal of IOL/capsulotomy & $1(0.5 \%)$ \\
Fragmatome lensectomy & $32(16 \%)$ \\
Fragmetome lensectomy/pupilloplasty & $2(1 \%)$ \\
Phacoemulsification & $9(5 \%)$ \\
Removal of silicone oil & $19(10 \%)$ \\
Removal of silicone oil/membranectomy & $1(0.5 \%)$ \\
Removal of silicone oil/pupilloplasty & $1(0.5 \%)$ \\
Epiretinal membrane peel & $8(4 \%)$ \\
Anterior vitrectomy & $3(1.5 \%)$ \\
Capsulotomy/capsulectomy & $3(1.5 \%)$ \\
\hline
\end{tabular}

Table 2 Reasons for >0.2LogMar visual acuity (VA) loss

\begin{tabular}{|c|c|}
\hline Reason for $>0.2 \log M a r V A$ loss & Number of patients \\
\hline Retinal detachment requiring further surgery & $3(25 \%)$ \\
\hline Branch retinal vein occlusion & $2(17 \%)$ \\
\hline Glaucoma & $2(17 \%)$ \\
\hline Corneal decompensation & $2(17 \%)$ \\
\hline Wet AMD & $1(8 \%)$ \\
\hline Diabetic maculopathy & $1(8 \%)$ \\
\hline Cystoid macular oedema & $1(8 \%)$ \\
\hline
\end{tabular}

causes of lens malposition included Weil-Marchesani syndrome $(n=1)$, homocystinuria $(n=1)$, familial ectopia lentis $(n=2)$, pseudoexfoliation syndrome $(n=1)$, and microspherophakia $(n=1)$.

\section{Previous surgery}

Four $(8 \%)$ of the patients with subluxed crystalline lenses had undergone previous vitreoretinal surgery before their lens subluxation. Of the patients with subluxed/ dislocated IOLs, 47 (40\%) had undergone at least one vitreoretinal procedure before their IOL displacement, 2 $(1.7 \%)$ had undergone trabeculectomy, and 1 (0.84\%) had a Baerveldt tube inserted. Of those patients who had had complicated cataract surgery, 15 (23\%) had undergone previous vitreoretinal surgery and $1(1.5 \%)$ had undergone previous trabeculectomy.

\section{Ocular comorbidities}

Coexisting, sight-threatening ocular morbidities, in addition to previous surgery, present before ACIOL insertion are shown in Table 4. 
Table 3 Reasons for ACIOL insertion together with their respective visual acuity (VA) changes

\begin{tabular}{lcc}
\hline Reason for ACIOL & Number of patients & Mean VA improvement (LogMar) (SD) (range) \\
\hline Subluxed/dislocated IOL & $118(44 \%)$ & $0.79(0.91)(-2.2$ to 2.68$)$ \\
Complicated cataract surgery & $65(24 \%)$ & $1.08(1.14)(-2.12$ to 2.7$)$ \\
Subluxed/dislocated crystalline lens & $45(17 \%)$ & $0.65(0.75)(-0.5$ to 2.52$)$ \\
Aphakia post previous VR surgery & $33(12 \%)$ & $0.32(0.96)(-1.8$ to 2.3$)$ \\
Aphakia post uncomplicated cataract surgery & $5(2 \%)$ & $0.3(0.28)(0-0.7)$ \\
Phacodonesis (trauma) & $3(1 \%)$ & $0.39(0.41)(0-0.82)$ \\
IOL exchange required & $2(1 \%)$ & $1.15(1.2)(0.3-2)$ \\
\hline
\end{tabular}

Table 4 Coexisting sight-threatening ocular morbidities

\begin{tabular}{lc}
\hline Coexisting ocular morbidity & Number of patients \\
\hline Previous trauma & $18(22 \%)$ \\
Glaucoma & $14(17 \%)$ \\
Uveitis/scleritis & $12(15 \%)$ \\
Proliferative diabetic retinopathy & $8(10 \%)$ \\
Age-related macular degeneration & $6(7 \%)$ \\
Amblyopia & $5(6 \%)$ \\
Retinitis pigmentosa & $4(5 \%)$ \\
Cystoid macula oedema & $3(4 \%)$ \\
Corneal pathology & $3(4 \%)$ \\
Macular hole/ERM & $3(4 \%)$ \\
Resolved choroidal detachment & $2(2 \%)$ \\
Optic neuropathy & $2(2 \%)$ \\
Retinal vascular malformation & $1(1 \%)$ \\
\hline
\end{tabular}

\section{Aciol size}

Three different ACIOL lengths were inserted: 12.5, 13, and $13.75 \mathrm{~mm}$. These were polymethylmethacrylate openloop angle-supported designs, manufactured by Bausch and Lomb Inc. (New York, NY, USA) (122UV lenses) (12.5 and $13.75 \mathrm{~mm}$ ) and Alcon (Hünenberg, Switzerland) (MTA-U0 lenses) (12.5 and $13 \mathrm{~mm}$ ). The number of patients receiving each of these different sized ACIOLs was: 70, 129, and 62 respectively. A white-to-white measurement (WTW), measured using the IOL master (Zeiss, Oberkochen, Germany), was recorded in the notes for 165 patients and the average difference between ACIOL length and WTW measurement was $0.98 \mathrm{~mm}$ (SD 0.5).

\section{Refractive outcomes}

Postoperative refractive outcome was available for 99 patients. Mean final spherical equivalent (SE) was $-0.39 \mathrm{D}$ (SD 1.5). The average difference between target refraction and final refraction was $-0.23 \mathrm{D}$ (SD 1.31).

\section{Postoperative complications}

Cystoid macular oedema Forty (15\%) patients developed postoperative $\mathrm{CMO}$. The rate of $\mathrm{CMO}$ did not differ significantly between those who were vitrectomised before ACIOL insertion (14\%) and those who were vitrectomised at the time (17\%) (two-sample $t$-test, $P=0.67)$. Thirteen of the 40 patients had a coexisting ocular comorbidity, such as intermediate uveitis or diabetic retinopathy, which may have predisposed them to the development of CMO. Thirty-three $(83 \%)$ patients were treated with topical steroid and/or a topical nonsteroidal anti-inflammatory drug alone. In addition to topical therapy, three patients required intravitreal triamcinolone acetonide, one patient required subTenon's triamcinolone acetonide, one patient required vitrectomy and epiretinal membrane peel, and two patients required oral prednisolone. Of the patients who were started on oral prednisolone, one patient continued to have persistent $\mathrm{CMO}$ at their most recent follow-up, which was thought to be due to sarcoid-related uveitis. The other patient's CMO resolved and they were on no medication at final follow-up.

Intraocular pressure rise Eighteen (7\%) patients were known to have glaucoma or ocular hypertension before ACIOL insertion. Eighty-seven (32\%) patients had an IOP rise above their preoperative levels acutely post ACIOL insertion. The rate of IOP rise did not differ significantly between those who were vitrectomised before ACIOL insertion (34\%) and those who were vitrectomised at the time $(31 \%)$ (two-sample $t$-test, $P=0.56)$. Sixty-one $(23 \%)$ patients remained on topical IOP lowering therapy at their most recent follow-up: 26 patients took 1 agent, 21 took 2, 13 took 3, and 1 took 4 . Five patients required glaucoma tube surgery, one had cyclodiode laser, and one had selective laser trabeculoplasty.

Corneal decompensation Fifteen (6\%) patients developed corneal decompensation after ACIOL insertion. Of these, five patients subsequently required corneal grafting: four patients underwent Descemet's stripping automated endothelial keratoplasty and one underwent penetrating keratoplasty. Patients who were followed up for longer were not more likely to get corneal decompensation than those who had a shorter follow-up (two-sample T-test, $P=0.42$ ). The rate of corneal decompensation did not differ significantly between those who were vitrectomised 
before ACIOL insertion ( $3 \%$ ) and those who were vitrectomised at the time ( $8 \%$ ) (two-sample $t$-test, $P=0.1$ ).

ACIOL displacement/instability Twenty (7\%) patients had ACIOL displacement and 14 (5\%) had ACIOL instability. Sixteen of these patients underwent further surgery: six patients had ACIOL exchange, three had ACIOL removal, and seven had ACIOL repositioning. In patients who had ACIOL displacement or instability, the ACIOL length minus WTW measurement was not significantly different from those patients who had no ACIOL displacement or instability (two-sample $T$-test, $P=0.12$ ). The rate of ACIOL displacement/instability did not differ significantly between those who were vitrectomised before ACIOL insertion (11\%) and those who were vitrectomised at the time (7\%) (two-sample $t$-test, $P=0.32$ ). Other postoperative complications are shown in Table 5 .

Hypotony/choroidal effusion occurred in four of the patients with leaking corneal wounds. Seven patients needed to return to the operating theatre for resuturing.

\section{Discussion}

Most recent studies looking at the outcome of ACIOL insertion have tended to look at outcomes following primary insertion after complicated cataract surgery/ intracapsular extraction ${ }^{7,8}$ or they have compared secondary scleral-fixated lenses to ACIOLs, thus limiting the numbers in the ACIOL arm. 2,3 This study, looking at a large cohort of patients with long follow-up, demonstrates how effective ACIOL insertion can be as a secondary procedure, after vitrectomy, in patients aphakic for multiple different reasons. Ninety-two per cent of our patients either maintained or gained visual acuity. This is a similar figure to the other studies that have looked at secondary ACIOL implantation after complicated cataract surgery. ${ }^{2,3}$ It is interesting that these figures are comparable given the large number of ocular comorbidities in our cohort and the many different reasons patients were aphakic.

The use of optical coherence tomography (OCT) to detect macular pathology has become more routine since the publication of previous studies looking at ACIOL outcomes. As a consequence, the incidence of $\mathrm{CMO}$ found in this study is likely to be more accurate than in previous studies. Despite this, a limitation of this study is that we did not perform OCT on every patient postoperatively, meaning our rate may be an underestimate of the true rate. Fifteen per cent of patients developed CMO in our study. This is, as expected, significantly more than the $0.1-2.35 \%{ }^{9}$ incidence that is quoted after routine cataract surgery and more comparable to that after extracapsular cataract extraction. ${ }^{10}$ It was reassuring that only seven
Table 5 Postoperative complications other than ACIOL instability, cystoid macular oedema, corneal decompensation, and raised intraocular pressure

\begin{tabular}{lc}
\hline Postoperative complication & Number of patients (\%) \\
\hline Retinal detachment & $15(6 \%)$ \\
Leaking corneal wounds/suture & $10(4 \%)$ \\
problems & \\
Acute anterior uveitis & $3(1 \%)$ \\
Branch retinal vein occlusion & $3(1 \%)$ \\
Monocular diplopia & $1(0.4 \%)$ \\
Acute angle closure & $1(0.4 \%)$ \\
\hline
\end{tabular}

patients required any more treatment for their $\mathrm{CMO}$ than topical therapy. This suggests that in the majority of patients after ACIOL placement, the $\mathrm{CMO}$ is acute and follows a similar course to $\mathrm{CMO}$ seen post phacoemulsification and lens implant surgery.

The increased IOP in 32\% of patients following surgery is of concern. Especially given the number of patients who continued to need pressure-lowering therapy in the long term. After ACIOL insertion in non-vitrectomised eyes the rate of IOP rise both in the short- and long-term varies greatly between studies. For example, one study found $100 \%$ of ACIOL patients had a long-term IOP rise, ${ }^{1}$ while another found only $4.1 \%$ of patients developed late secondary glaucoma. ${ }^{2}$ Given this wide range, it is difficult to say whether an IOP rise is more likely in a vitrectomised eye or not. It is possible to conclude, however, that patients need to be properly counselled before ACIOL insertion about the risks of raised pressure and IOP needs careful monitoring after surgery.

The optimum diameter of an ACIOL is thought to be 0.5-1.0 mm greater than the horizontal WTW. ${ }^{11}$ Despite the average difference between ACIOL length and WTW measurement being $0.93 \mathrm{~mm}$ in our series, we still had several cases with ACIOL instability/displacement.

The average difference between target refraction and final refraction was $-0.23 \mathrm{D}$. This compares favourably to previous studies ${ }^{12}$ and reinforces the idea that with ACIOL placement postvitrectomy we should expect to achieve refractive outcomes comparable to posterior chamber IOL placement.

Corneal decompensation has been a theoretical concern with ACIOL placement since they were first introduced. The relatively low number of patients developing decompensation in this study is similar to that in nonvitrectomised eyes, ${ }^{2,4,5,7}$ and is reassuring that with openloop anterior chamber lenses corneal problems only rarely occur, even in patients who have had multiple previous vitreoretinal procedures. Given that some patients had only 3 months of follow-up, a limitation of this study is that we may have underestimated the true rate of corneal decompensation. However, the fact that patients who had longer follow-up were not significantly more likely to get 
corneal decompensation than those with a short followup suggests that this was not a significant limitation.

In conclusion, this is the first study to look specifically at secondary ACIOL insertion in a large number of vitrectomised eyes aphakic for multiple different reasons. It demonstrates that ACIOL placement is effective and has an acceptable risk profile in often complex cases. ACIOLs generally achieve good visual acuity and refractive results post vitrectomy.

\section{Summary}

\section{What was known before}

- ACIOLs can be an effective means of treating aphakia in non-vitrectomised eyes, but there are recognised complications associated with them

\section{What this study adds}

- Anterior chamber lenses are an effective means of treating aphakia in vitrectomised eyes.

- Complication rates are relatively low.

\section{Conflict of interest}

The authors declare no conflict of interest.

\section{Acknowledgements}

This research was supported by the NIHR Biomedical Research Centre at Moorfields Eye Hospital NHS Foundation Trust and UCL Institute of Ophthalmology and the NIHR Moorfields Clinical Research Facility. The views expressed are those of the authors and not necessarily those of the NHS, the NIHR, or the Department of Health.

\section{References}

1 Kwong YY, Yuen HKL, Lam RF, Lee VY, Rao SK, Lam DS. Comparison of outcomes of primary scleral-fixated versus primary anterior chamber intraocular lens implantation in complicated cataract surgery. Ophthalmology 2007; 114: 80-85.

2 Evereklioglu C, Er H, Bekir NA, Borazan M, Zorlu F. Comparison of secondary implantation of flexible open-loop anterior chamber and scleral-fixated posterior chamber intraocular lenses. J Cataract Refract Surg 2003; 29: 301-308.

3 Bellucci R, Pucci V, Morselli S, Bonomi L. Secondary implantation of angle-supported anterior chamber and scleral-fixated posterior chamber intraocular lenses. J Cataract Refract Surg 1996; 22: 247-252.

4 Donaldson KE, Gorscak JJ, Budenz DL, Feuer WJ, Benz MS, Forster RK. Anterior chamber and sutured posterior chamber intraocular lenses in eyes with poor capsular support. J Cataract Refract Surg 2005; 31: 903-909.

5 Chan TC, Lam JK, Jhani V, Li EY. Comparison of outcomes of primary anterior chamber versus secondary scleral-fixated intraocular lens implantation in complicated cataract surgeries. Am J Ophthalmol 2015; 159: 221-226.

6 Auffarth GU, Wesendahl TA, Brown SJ, Apple DJ. Are there acceptable anterior chamber intraocular lenses for clinical use in the 1990s? Ophthalmology 1994; 101: 1913-1922.

7 Brunin G, Khandelwal S, Coffee R, Orengo-Nania S, Biggerstaff KS. Outcomes of anterior chamber intraocular lenses placed by surgeons in training. J Cataract Refract Surg 2015; 41: 2102-2106.

8 Hennig A, Evans JR, Pradhan D, Johnson GJ, Pokhrel RP, Gregson RM et al. Randomised controlled trial of anteriorchamber intraocular lenses. Lancet 1997; 349: 1129-1133.

9 Lowenstein A, Zur D. Postsurgical cystoid macular edema. Dev Ophthalmol 2010; 47: 149-159.

10 Flach AJ. The incidence, pathogenesis and treatment of cystoid macular edema following cataract surgery. Trans Am Ophthalmol Soc 1998; 96: 667-634.

11 Lovisolo CF, Reinstein DZ. Phakic intraocular lenses. Surv Ophthalmol 2005; 50: 549-587.

12 Gore DM, Wilkins MR. Refractive outcomes in anterior chamber intraocular lenses. Int Ophthalmol 2013; 33: 453-454. 